\title{
Two galatheid associates of crinoids from the Ryukyu Islands (Decapoda: Anomura: Galatheidae), with their ecological notes
}

Yoshihisa Fujita and Keiji Baba

\begin{abstract}
Galathea amboinensis De Man, 1888 and G. inflata Potts, 1915, both known as associates of shallow water comatulid crinoids, are reported for the first time from Japan. In the Ryukyu Islands, Galathea inflata was found on 13 species of crinoids but most commonly on Comanthus parvicirrus (Müller) and Comaster schlegelii (Carpenter). Galathea amboinensis was recorded from 10 crinoid species but more often on Capillaster multiradiatus (Linnaeus) than on other crinoids. Heterosexual pairs of $G$. inflata were found on $26.3 \%$ of host crinoids $(n=57)$, and all females were ovigerous. Megalopae of both galatheids were found on hosts, indicating that the larvae settled on crinoids at the megalopa stage.
\end{abstract}

\section{Introduction}

In 1995, one of us (YF) collected some unusual galatheids on crinoids during studies of the crinoid fauna of the Ryukyu Islands. These galatheids were then identified by KB as Galathea amboinensis de Man, 1888 and G. inflata Potts, 1915. Galathea amboinensis had been recorded from Ambon, Torres Strait, Moluccas and Sulu Archipelago and G. inflata from Torres Strait and the Molucca Islands, both as associates of comatulid crinoids (see Baba, 1979, 1988). Since then special attention has been paid to host specificity of these two galatheids, by collecting as many crinoids as possible. A total of 543 crinoids distributed among 36 species have been collected, and 108 specimens of the two galatheids have been obtained from 80 of the crinoids collected. The material at hand now provides new information on color in life, host preference, stage of settlement on hosts, and size in relation to sex determination of the galatheids.

The collections and observations were conducted by YF in coastal waters of Okinawa Island (five locations on the west coast: Sesoko, Motobu-cho; Cape Maeda, Onna-son; Udui, Onna-son; Mizugama, Kadena-cho; and Sunabe, Chiatancho) by SCUBA diving during the day and night. Each crinoid was collected by enclosing it in a plastic bag that prevented the escape of any crustacean associates. The crinoids were identified with the help of Dr. Ichizo Kogo, a specialist of crinoid taxonomy, and by consultation with the following papers: Clark (1931, 1941, 1947), Clark \& Rowe (1971), Hoggett \& Rowe (1986), Kogo (1998), Messing (1998), Rowe et al. (1986), and Utinomi \& Kogo (1965, 1968). The postorbital carapace length (CL) indicates size of the galatheids.

\section{Galathea amboinensis De Man, 1888} Fig. 1

\section{Material examined.-See Table 1.}

Recognition characters.-Carapace with lateral margins strongly convex, bearing 5 spines behind anterior cervical groove, gastric region without spines, anterior branchial region with continuous ridges. Epipods present on chelipeds and first two walking legs. Third maxilliped 
Table 1. The material examined of Galathea amboinensis. Indet. = indeterminate; FG $=$ female gonopores present; $\mathrm{SP}=$ short pleopods.

\begin{tabular}{|c|c|c|c|c|c|}
\hline Date & locality & Depth (m) & Host & Sex & Size \\
\hline 26.04.1996 & Cape Maeda & 7.3 & Capillaster multiradiatus & Indet. (FG, SP) & 2.70 \\
\hline 26.04.1996 & Cape Maeda & 7.2 & Capillaster multiradiatus & Indet. (FG, SP) & 2.25 \\
\hline \multirow[t]{3}{*}{ 12.03.1997 } & Cape Maeda & 6.3 & Capillaster multiradiatus & Megalopa & 0.99 \\
\hline & & & & Indet. $(\mathrm{SP})^{1)}$ & 1.39 \\
\hline & & & & Indet. (FG, SP) & 3.80 \\
\hline \multirow[t]{3}{*}{12.03 .1997} & Cape Maeda & 5.5 & Capillaster multiradiatus & Megalopa & 0.99 \\
\hline & & & & Megalopa & 0.92 \\
\hline & & & & Megalopa & 0.99 \\
\hline \multirow[t]{2}{*}{28.03 .1997} & Cape Maeda & 8.7 & Capillaster multiradiatus & Megalopa & 0.86 \\
\hline & & & & Megalopa & 1.12 \\
\hline \multirow[t]{2}{*}{28.03 .1997} & Cape Maeda & 6.8 & Capillaster multiradiatus & Indet. (FG, SP) & 5.60 \\
\hline & & & & Indet. (FG, SP) & 4.50 \\
\hline 28.03.1997 & Cape Maeda & 7.1 & Capillaster multiradiatus & Megalopa & 1.06 \\
\hline 28.03.1997 & Cape Maeda & 5.9 & Capillaster multiradiatus & Indet. (FG, SP) & 1.80 \\
\hline 28.03.1997 & Cape Maeda & 7.1 & Capillaster multiradiatus & Megalopa & 1.06 \\
\hline 03.04.1997 & Cape Maeda & 8.1 & Capillaster multiradiatus & Indet. $(\mathrm{SP})^{1)}$ & 1.30 \\
\hline 01.12 .1997 & Mizugama & 7.1 & Comanthus gisleni & Indet. (FG, SP) & 4.60 \\
\hline \multirow[t]{2}{*}{ 09.07.1997 } & Cape Maeda & 5.9 & Comanthus parvicirrus & Indet. (FG, SP) & 1.85 \\
\hline & & & & Indet. (FG, SP) & 1.25 \\
\hline 11.09.1997 & Cape Maeda & 22.5 & Comanthus sp. & Indet. (FG, SP) & 1.80 \\
\hline 21.07.1996 & Mizugama & 6.8 & Comaster nobilis & Indet. (FG, SP) & 3.50 \\
\hline 21.07.1996 & Mizugama & 6.8 & Comaster nobilis & ov. $q$ & 5.50 \\
\hline 12.05 .1995 & Mizugama & 9.0 & Comaster nobilis & Indet. (FG, SP) & 2.18 \\
\hline 16.08 .1995 & Cape Maeda & 12.5 & Comaster nobilis & Megalopa & 1.03 \\
\hline 03.06.1999 & Udui & 29.8 & Comatella nigra & Indet. (FG, SP) & 3.20 \\
\hline \multirow[t]{2}{*}{29.04 .1997} & Cape Maeda & 7.1 & Dichrometra sp. & Megalopa & 1.05 \\
\hline & & & & Indet. (FG, SP) & 1.55 \\
\hline 03.08.1996 & Mizugama & 7.8 & Phanogenia gracilis & Indet. (FG, SP) & 2.90 \\
\hline 14.10.1996 & Mizugama & 9.7 & Phanogenia gracilis & ov. $q$ & 7.28 \\
\hline \multirow[t]{2}{*}{29.04 .1997} & Cape Maeda & 8.2 & Stephanometra spicata & Indet. (FG, SP) & 2.80 \\
\hline & & & & Megalopa & 0.99 \\
\hline 12.05 .1995 & Mizugama & 7.3 & Tropiometra afra macrodiscus & Indet. (FG, SP) & 2.30 \\
\hline
\end{tabular}

1) Presence or absence of female gonopres was not determined because of poor conditions of the specimen.

ischia with $15-17$ denticles on mesial ridge, merus with two strong spines on flexor margin and 1 distal spine on extensor margin. Chelipeds spinose and short relative to their width, carpus at most twice as long as broad.

Coloration.-Dark brown and graywhite stripes on carapace and abdomen; 

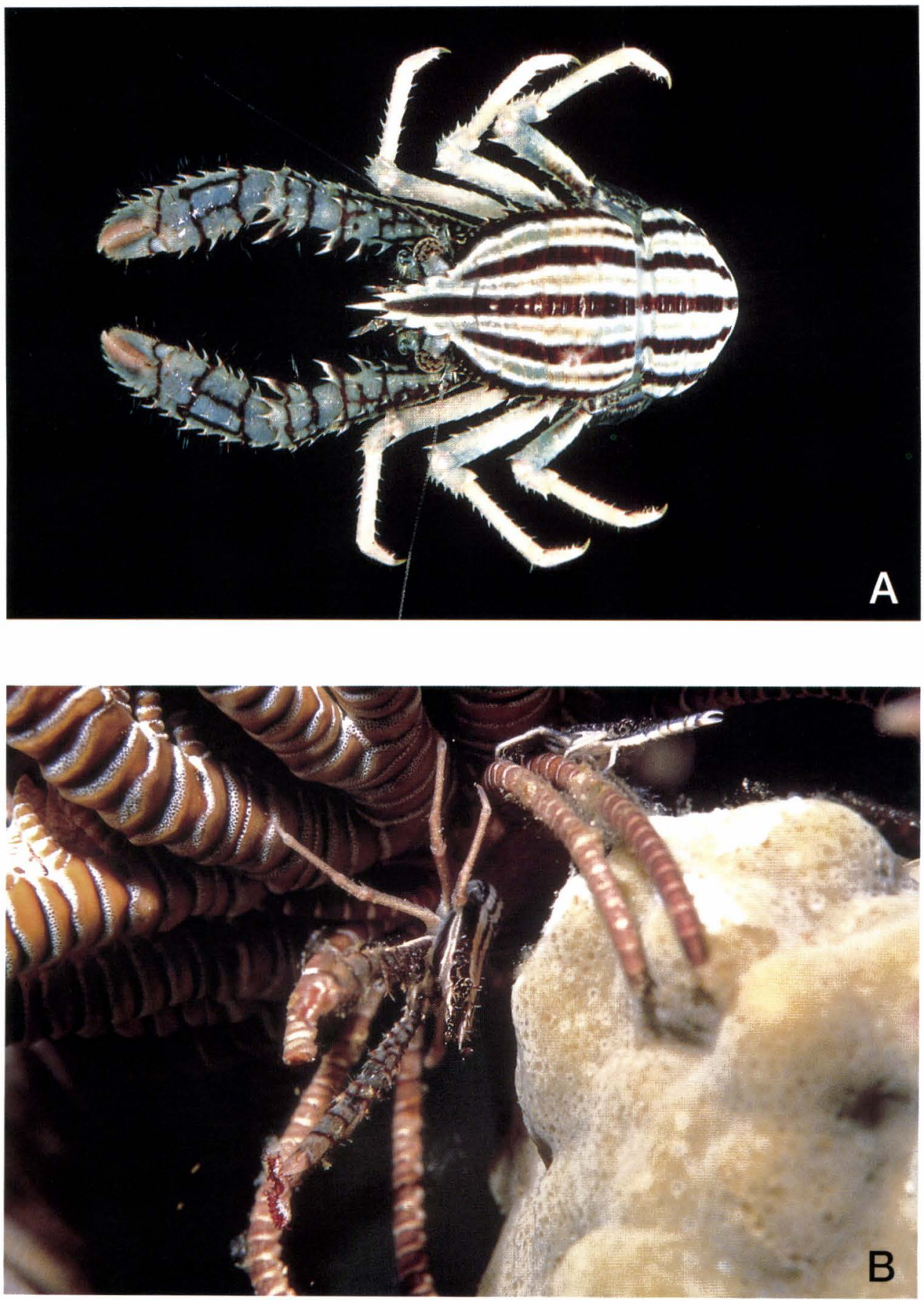

Fig. 1. Galathea amboinensis De Man: A, ovigerous female $(\mathrm{CL}=7.28 \mathrm{~mm})$, collected from Phanogenia gracilis (Hartlaub); B, living posture at night, associated with Capillaster multiradiatus (Linnaeus). Photos taken by Y. Fujita. 
chelipeds gray-white, with dark brown bands; walking legs whitish (Fig. 1A).

Hosts.-The present material was associated with 10 species of crinoids (Tables 1, 3): Capillaster multiradiatus (Linnaeus), Phanogenia gracilis (Hartlaub), Comatella nigra (Carpenter), Comaster nobilis (Carpenter), Comanthus parvicirrus (Müller), C. gisleni Rowe et al., Comanthus sp. 1, Dichrometra sp., Stephanometra spicata (Carpenter), and Tropiometra afra macrodiscus (Hara).

Potts (1915) recorded Comanthus annulatum as a host of $G$. amboinensis. By consultation with Clark (1915, 1931) and Rowe et al. (1986), this comatulid may now be referable to $C$. parvicirrus, but its systematic status is still unclear. Also, "Comanthina schlegeli," another host reported by Baba (1979), has recently been divided into two species, Comaster schlegelii (Carpenter) and Comaster nobilis (Carpenter) (see Rowe et al., 1986; Messing, 1998). Thus, at least seven (excluding $C$. nobilis, C. parvicirrus, and Comanthus sp. 1) of the host crinoids listed above can be recorded here as new hosts.

Range.-Previously recorded from Ambon (type locality), Torres Strait, Moluccas and Sulu Archipelago (de Man, 1888; Potts, 1915; Baba, 1979, 1988). The present material constitutes the first record of $G$. amboinensis from Japan.

Ecological notes.-Galathea amboinensis is found between the cirri and basal parts of the arms of crinoids such as Capillaster multiradiatus, Comatella nigra, Dichrometra sp., Stephanometra spicata and Tropiometra afra macrodiscus, which bear well developed cirri (Fig. 1B). When host crinoids have cirri reduced or absent (Phanogenia gracilis, Comanthus gisleni, C. parvicirrus, C. sp. 1 , and Comaster nobilis), the galatheids are found on substrate under the host. Night observations revealed that $G$. amboinensis often ventured slightly away from the host.
The galatheids found on 16 of 23 host crinoids were solitary, and two or three individuals (mostly megalopae and juveniles of undetermined sex) were found on each of the remaining 7 hosts. No heterosexual pairs were found. No males were collected and all females were ovigerous.

Frequency of occurrence of the galatheids on each host species is shown in Table 3. Capillaster multiradiatus housed more than half the total number of the galatheids collected.

Co-inhabitants on the crinoids include: Palaemonella pottsi (Borradaile), Parapontonia nudirostris Bruce, Periclimenes affinis (Zehantner), P. amboinensis (De Man), P. ceratophthalmus Borradaile, $P$. commensalis Borradaile, $P$. pilipes Bruce \& Zmarzly, Pontoniopsis comanthi Borradaile, Synalpheus demani Borradaile, Synalpheus stimpsoni (De Man), Synalpheus sp., Galathea inflata, Harrovia longipes Lanchester, and Permanotus purpureus (Gordon). Further details will be reported elsewhere (Fujita, unpublished).

Measurements.-Megalopae, CL = 0.86-1.12 mm; sex indeterminate (female gonopores present, pleopods short), $\mathrm{CL}=1.30-5.60 \mathrm{~mm}$; ovigerous females, $\mathrm{CL}=5.5,7.28 \mathrm{~mm}$.

\section{Galathea inflata Potts, 1915}

\section{Fig. 2}

\section{Material examined.-See Table 2.}

Recognition characters.- Carapace lateral margins convex, bearing 6 spines behind anterior cervical groove; no gastric spines; anterior branchial region with scale-like ridges. Epipods present on chelipeds and first and second walking legs. Ischium of third maxilliped with more than 23 denticles on mesial ridge; merus with 3 spines on flexor margin, 2 spines on extensor margin. Chelipeds long relative to their width, carpus more than twice as long as broad. 
Table 2. The material examined of Galathea inflata. Indet. = sex indeterminate; FG = female gonopores present; No FG = Female gonopores absent; $\mathrm{SP}=$ short pleopods.

\begin{tabular}{|c|c|c|c|c|c|}
\hline Date & locality & Depth (m) & Host & Sex & Size \\
\hline 12.03 .1997 & Cape Maeda & 6.7 & Capillaster multiradiatus & Megalopa & 1.15 \\
\hline 12.03 .1997 & Cape Maeda & 5.1 & Capillaster multiradiatus & Indet. (No FG, SP) & 1.40 \\
\hline 28.03 .1997 & Cape Maeda & 6.2 & Capillaster multiradiatus & Megalopa & 1.16 \\
\hline \multirow[t]{2}{*}{ 28.03.1997 } & Cape Maeda & 6.7 & Capillaster multiradiatus & Megalopa & 1.20 \\
\hline & & & & Indet. (FG, SP) & 1.85 \\
\hline 02.04.1997 & Cape Maeda & 6.2 & Capillaster multiradiatus & Indet. (FG, SP) & 2.30 \\
\hline 02.04.1997 & Cape Maeda & 6.3 & Capillaster multiradiatus & Indet. (No FG, SP) & 1.40 \\
\hline 02.04.1997 & Cape Maeda & 5.5 & Capillaster multiradiatus & Indet. (No FG, SP) & 1.50 \\
\hline \multirow[t]{2}{*}{ 03.04.1997 } & Cape Maeda & 6.9 & Capillaster multiradiatus & Indet. (No FG, SP) & 2.00 \\
\hline & & & & ov. $q$ & 3.50 \\
\hline 03.04.1997 & Cape Maeda & 7.9 & Capillaster multiradiatus & $q$ & 4.62 \\
\hline 10.01.1997 & Mizugama & 6.2 & Clarkcomanthus littoralis & $q$ & 3.80 \\
\hline 12.05 .1997 & Cape Maeda & 28.0 & Comanthus alternans & Indet. (No FG, SP) & 1.85 \\
\hline \multirow[t]{2}{*}{03.08 .1996} & Mizugama & 9.7 & Comanthus parvicirrus & ov. $q$ & 5.90 \\
\hline & & & & $\delta$ & 3.30 \\
\hline \multirow[t]{2}{*}{ 15.11.1996 } & Mizugama & 8.0 & Comanthus parvicirrus & $\delta$ & 3.10 \\
\hline & & & & ov. $q$ & 5.80 \\
\hline 21.11.1996 & Mizugama & 8.4 & Comanthus parvicirrus & ㅇ & 6.00 \\
\hline 10.01.1997 & Mizugama & 7.4 & Comanthus parvicirrus & ov. $q$ & 5.40 \\
\hline 30.01.1997 & Mizugama & 6.8 & Comanthus parvicirrus & $\delta$ & 5.20 \\
\hline \multirow[t]{2}{*}{ 20.03.1997 } & Mizugama & 7.6 & Comanthus parvicirrus & $\delta$ & 4.20 \\
\hline & & & & ov. $q$ & 3.80 \\
\hline 20.03.1997 & Mizugama & 6.1 & Comanthus parvicirrus & $\delta$ & 4.20 \\
\hline 25.03.1997 & Mizugama & 6.2 & Comanthus parvicirrus & 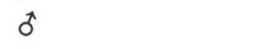 & 3.36 \\
\hline 03.04.1997 & Cape Maeda & 7.6 & Comanthus parvicirrus & q & 5.32 \\
\hline \multirow[t]{2}{*}{ 24.06.1999 } & Cape Maeda & 6.8 & Comanthus parvicirrus & ov. $q$ & 3.70 \\
\hline & & & & 0 & 5.10 \\
\hline 12.06.1999 & Cape Maeda & 5.2 & Comanthus parvicirrus & Indet. (FG, SP) & 1.85 \\
\hline \multirow[t]{2}{*}{ 13.04.1997 } & Mizugama & 5.5 & Comanthus parvicirrus & $\sigma^{\pi}$ & 4.62 \\
\hline & & & & ov. $q$ & 5.32 \\
\hline \multirow[t]{2}{*}{ 21.04.1997 } & Mizugama & 7.6 & Comanthus parvicirrus & t & 3.78 \\
\hline & & & & ov. 9 & 5.18 \\
\hline 21.04.1997 & Mizugama & 4.9 & Comanthus parvicirrus & Indet. (No FG, SP) & 1.10 \\
\hline 14.05.1997 & Mizugama & 8.0 & Comanthus parvicirrus & Indet. (FG, SP) & 1.60 \\
\hline \multirow[t]{2}{*}{ 27.08.1997 } & Sesoko & 3.2 & Comanthus parvicirrus & ov. $q$ & 5.60 \\
\hline & & & & ot & 3.30 \\
\hline \multirow[t]{2}{*}{ 31.08.1997 } & Sesoko & 5.7 & Comanthus parvicirrus & Megalopa & 0.96 \\
\hline & & & & o & 5.60 \\
\hline \multirow[t]{2}{*}{31.08 .1997} & Sesoko & 6.1 & Comanthus parvicirrus & Indet. (No FG, SP) & 1.75 \\
\hline & & & & o & 3.30 \\
\hline \multirow{3}{*}{31.08 .1997} & & & & ov. $q$ & 4.40 \\
\hline & Sesoko & 6.1 & Comanthus parvicirrus & $\delta$ & 5.18 \\
\hline & & & & ov. $\&$ & 5.60 \\
\hline \multirow[t]{2}{*}{31.08 .1997} & Sesoko & 6.3 & Comanthus parvicirrus & $\delta$ & 4.48 \\
\hline & & & & ov. $q$ & 5.74 \\
\hline 12.10 .1997 & Mizugama & 7.4 & Comanthus sp & $q$ & 3.30 \\
\hline 26.08.1997 & Sesoko & 9.8 & Comaster nobilis & $\sigma$ & 2.40 \\
\hline 27.04.1996 & Mizugama & 2.0 & Comaster schlegelii & 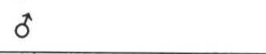 & 4.40 \\
\hline
\end{tabular}


Table 2. Continued.

\begin{tabular}{|c|c|c|c|c|c|}
\hline Date & locality & Depth (m) & Host & Sex & Size \\
\hline \multirow[t]{2}{*}{ 27.12.1996 } & Mizugama & 6.7 & Comaster schlegelii & ov. $q$ & 5.70 \\
\hline & & & & $\delta$ & 4.40 \\
\hline 07.01 .1997 & Mizugama & 8.1 & Comaster schlegelii & $\hat{0}$ & 5.90 \\
\hline \multirow[t]{2}{*}{30.01 .1997} & Mizugama & 4.8 & Comaster schlegelii & $\hat{0}$ & 4.00 \\
\hline & & & & ov. 우 & 4.70 \\
\hline 30.01 .1997 & Mizugama & 5.1 & Comaster schlegelii & 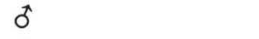 & 3.50 \\
\hline 13.04.1997 & Mizugama & 5.6 & Comaster schlegelii & 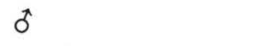 & 5.40 \\
\hline 21.04.1997 & Mizugama & 4.7 & Comaster schlegelii & ov. +9 & 5.04 \\
\hline 21.04.1997 & Mizugama & 6.2 & Comaster schlegelii & Indet. (No FG, SP) & 1.75 \\
\hline 21.04.1997 & Mizugama & 4.7 & Comaster schlegelii & Indet. (FG, SP) & 1.50 \\
\hline \multirow[t]{2}{*}{ 22.04.1997 } & Mizugama & 5.2 & Comaster schlegelii & $\sigma$ & 5.32 \\
\hline & & & & ov. 9 & 5.60 \\
\hline 22.04.1997 & Mizugama & 6.0 & Comaster schlegelii & $\delta$ & 5.60 \\
\hline \multirow[t]{2}{*}{ 22.04.1997 } & Mizugama & 5.5 & Comaster schlegelii & $\delta$ & 5.60 \\
\hline & & & & ov. + & 6.30 \\
\hline 13.10.1997 & Mizugama & 6.9 & Comatella maculata & ov. +9 & 4.30 \\
\hline 12.10 .1997 & Mizugama & 8.3 & Comatella nigra & $q$ & 3.70 \\
\hline 15.11.1996 & Mizugama & 7.8 & Comatella stelligera & $\delta$ & 3.90 \\
\hline 29.08.1996 & Mizugama & 5.5 & Phanogenia gracilis & Indet. (No FG, SP) & 2.30 \\
\hline \multirow[t]{2}{*}{ 14.11.1997 } & Mizugama & 6.6 & Phanogenia gracilis & ov. $q$ & 5.30 \\
\hline & & & & $\delta$ & 4.20 \\
\hline 02.04.1997 & Cape Maeda & 4.4 & Stephanometra spicata & Indet. (No FG, SP) & 1.40 \\
\hline 09.09.1997 & Cape Maeda & 6.1 & Stephanometra spicata & Megalopa & 1.05 \\
\hline 07.01 .1997 & Mizugama & 9.0 & Tropiometra afra macrodiscus & Indet. (No FG, SP) & 1.10 \\
\hline 30.04 .1995 & Mizugama & 6.6 & Tropiometra afra macrodiscus & Indet. (No FG, SP) & 1.45 \\
\hline 12.05.1995 & Mizugama & 8.1 & Tropiometra afra macrodiscus & Indet. (No FG, SP) & 1.58 \\
\hline 12.05.1995 & Mizugama & 7.3 & Tropiometra afra macrodiscus & Indet. (No FG, SP) & 1.65 \\
\hline 25.07.1995 & Sunabe & 7.6 & Tropiometra afra macrodiscus & ov. + & 3.40 \\
\hline
\end{tabular}

Coloration.-Two color patterns were recognized in the material examined:

Color pattern 1 (Fig. 2A): Body dark brown ground color, with 2 yellow- white stripes on carapace extending backward to join each other on abdomen; chelipeds dark brown, bearing white spot on tip of each finger; walking legs also dark brown, with yellow-white band on distal portion of propodus, dactyls distally whitish.

Color pattern 2 (Fig. 2B): Very similar to pattern 1 but ground color dark green. This coloration was seen only on specimens from Comaster schlegelii, the ventral side of which is green.

Hosts.-The present material was found in association with the following 13 species of crinoids: Capillaster multiradiatus (Linnaeus), Phanogenia gracilis (Hartlaub), Comatella maculata (Carpenter), Comatella nigra (Carpenter), Comatella stelligera (Carpenter), Comanthus alternans (Carpenter), Comanthus parvicirrus (Müller), Comanthus sp. 1, Comaster nobilis (Carpenter), Comaster schlegelii (Carpenter), Clarkcomanthus littoralis (Carpenter), Stephanometra spicata (Carpenter) and Tropiometra afra macrodiscus (Hara) (see Table 3).

The type material was known from "Comanthus annulatum" (see Potts, 1915; see above under G. amboinensis for systematic status of this host). Baba (1979) reported that the Moluccan material was 


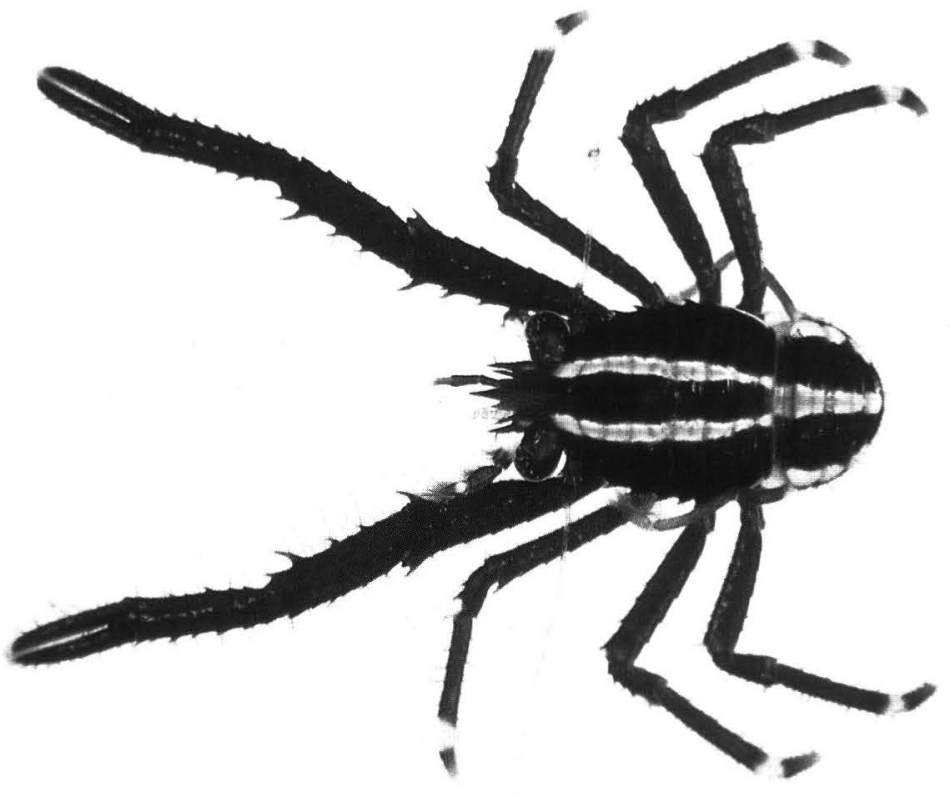

A

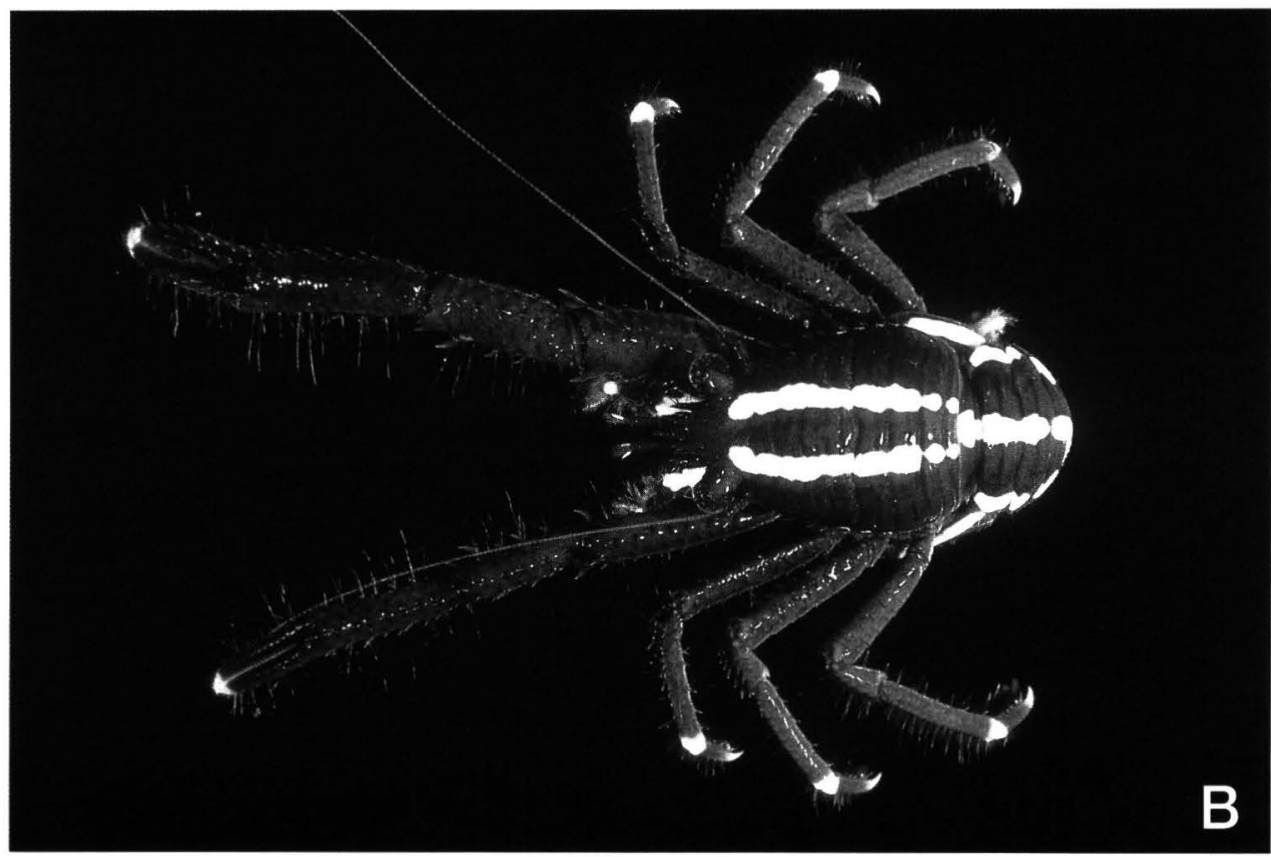

Fig. 2. Galathea inflata Potts: A, ovigerous female $(\mathrm{CL}=3.80 \mathrm{~mm})$, collected from Comanthus parvicirrus (Müller); B. male $(\mathrm{CL}=5.90 \mathrm{~mm})$, collected from Comaster schlegelii (Carpenter). Photos taken by Y. Fujita. 
found on Comanthus bennetti (now called Oxycomanthus bennetti), Comanthus parvicirrus and Comanthina schlegeli (= Comaster schlegelii or C. nobilis; see above). At least eight species of the crinoids (excluding C. parvicirrus, Comanthus sp. 1, C. nobilis, C. schlegelii, C. littoralis) listed above apparently represent new hosts.

Range.-Previously known from Torres Strait (type locality; Potts, 1915) and the Molucca Islands (Baba, 1979). Galathea inflata is recorded from Japanese waters for the first time.

Ecological notes.-Like G. amboinensis, $G$. inflata was found on crinoids irrespective of the character of the cirri of the host-well developed or reduced. When associated with C. parvicirrus and C. schlegelii that usually live in crevices on coral reefs, the galatheids were often found on the substrate.

Galathea inflata was most commonly found on $C$. parvicirrus (the galatheids were found on 20 of $31=64.5 \%$ of crinoids collected) and taken on 12 of $34=35.3 \%$ of C. schlegelii collected (Table 3 ).

Heterosexual pairs were found on 15 of 57 host crinoids: on 10 of 20 C. parvicirrus, 4 of 12 C. schlegelii, and 1 of 2 Phanogenia gracilis. The females of all heterosexual pairs were ovigerous and mostly larger than their accompanying males. The remaining hosts were mostly occupied by single galatheids including males, ovigerous females, non-ovigerous females, juveniles (sex indeterminate) and megalopae. Two other pairs were found: an ovigerous female and juvenile (sex indet., bearing no female gonopores); one megalopa and juvenile (sex indet., bearing female gonopores).

Co-inhabitants found on the crinoids were: Palaemonella pottsi, Parapontonia nudirostris, Periclimenes affinis, $P$. amboinensis, $P$. ceratophthalmus, $P$. commensalis, Pontoniopsis comanthi, Synalpheus demani, Synalpheus stimpsoni, Synalpheus sp., Galathea amboinensis and Permanotus purpureus. More detail will be reported later elsewhere (Fujita, unpublished).

Measurements.-Megalopae, $\mathrm{CL}=0.96-1.20 \mathrm{~mm}$; incomplete males (no genital pores on second walking legs, no gonopods), $\mathrm{CL}=1.10-2.30 \mathrm{~mm}$; incomplete females (gonopores on second walking legs, no female pleopods), $\mathrm{CL}=1.50-2.30$ $\mathrm{mm}$; males, $\mathrm{CL}=2.40-5.90 \mathrm{~mm}$; females, $\mathrm{CL}=3.30-6.30 \mathrm{~mm}$ (ovigerous females from $\mathrm{CL}=3.40 \mathrm{~mm}$ ).

\section{Discussion}

Using the numerous records of crinoid symbionts in the literature, Potts (1915) was the first to focus upon crustaceancrinoid associations, in which the present two species of galatheids were included. These associations have also been discussed by Fishelson (1974), Zmarzly (1984), and Fabricius \& Dale (1993), based upon material from shallow waters in the Red Sea, Great Barrier Reef, and Eniwetok Atoll. So far as the shallow water galatheid symbionts are concerned, four species are known: Allogalathea elegans (Adams \& White, 1848) (see Potts, 1915; Barnard, 1950; Holthuis, 1953; Lewinsohn, 1969; Baba, 1979), Galathea amboinensis (see Potts, 1915, under G. minuta Potts; Baba, 1979), Galathea genkai Miyake \& Baba, 1966 (see Lewinsohn, 1969; Fishelson, 1974), and Galathea inflata (see Potts, 1915; Baba, 1979). Miyake (1938) noted that Galathea acanthomera Stimpson, 1858 (=now G. orientalis Stimpson, 1858) was taken from a crinoid. Utinomi \& Kogo (1965) reported $G$. orientalis and $G$. sp. as associates of crinoids. However, $G$. orientalis is usually found to be free living in Japanese coastal waters. Fabricius \& Dale (1993) listed three unidentified species of Galathea that they found on crinoids from the Great Barrier Reef. Eeckhaut et al. (1998) reported two spe- 
cies of galatheids (without giving scientific names) on crinoids from Papua-New Guinea.

\section{Host specificity}

Our study shows that Galathea amboinensis is associated with 10 species and $G$. inflata with 13 species of crinoids. Compared with the pontoniine Periclimenes novaecaledoniae and the alpheid Synalpheus demani, both known from single crinoids (Bruce, 1982), host specificity of these galatheids is rather low. This is also true for Allogalathea elegans, which has been recorded from nine species of crinoids (Baba, 1979). However, other galatheids are more specialized: Galathea genkai is known from three crinoid hosts (see above for references), and a deep-sea species of the galatheid genus Munidopsis is known from two crinoids (Rice \& Miller, 1992).

Although 15 host crinoid species are known in the Ryukyu Islands, colonization rate (indicated by percent occurrence, i.e. the number of hosts found by the galatheids) of Galathea inflata is relatively high for Comanthus parvicirrus $(64.5 \%, \mathrm{n}=31)$ and Comaster schlegelii (35.3\%, $\mathrm{n}=34$ ), while that of $G$. amboinensis is highest for Capillater multiradiatus (21.7\%, $\mathrm{n}=46$ ). Zmarzly (1984) noted that $54 \%(\mathrm{n}=13)$ of Comanthina schlegelii (= now Comaster schlegelii) housed Allogalathea elegans, as also did $23 \%(n=73)$ of Comanthus bennetti (= now Oxycomanthus bennetti). Fabricius \& Dale (1993) reported that the species of Galathea and Allogalathea they observed were not found on those crinoids that lack cirri. This does not seem to be always true, because both the species we report here have been taken from hosts having reduced cirri. In addition, at least one of the previously reported host crinoids for $A$. elegans (C. parvicirrus) has reduced cirri.

Potts (1915) reported that galatheids leave their host when disturbed but when they have been detached they swim back immediately to the host.

\section{Sex determination in relation to size}

Our study is the first to report that galatheid larvae settle on crinoids at the megalopa stage (Tables 1, 2).

From the megalopa Galathea inflata grows into one of two sexually indeterminate forms (juveniles): one having the second walking legs with gonopores but with short pleopods ( $\mathrm{CL}=1.50-2.30 \mathrm{~mm})$, and the other lacking both female gonopores and male gonopods $(\mathrm{CL}=1.10-2.30 \mathrm{~mm})$. Subsequently they attain sizes that allow discrimination of their sex at $\mathrm{CL}=2.40$ $\mathrm{mm}$ for males and $3.30 \mathrm{~mm}$ for females. The male is determined by the presence of a pair of gonopods on each of the first and second abdominal segments, and the female by the presence of gonopores on the coxae of the second walking legs and elongate, biramous pleopods. Dr. Yasushi Fukuda, Kyushu Luther College, Kumamoto reared larvae of Galathea orientalis Stimpson, 1858, up to the fourth crab stage under laboratory conditions. He found that it was at the second stage that female gonopores became discernible but the pleopods were not typical of the sexes through the fourth crab stage (Fukuda, unpublished). The size distribution displayed by $G$. inflata suggests that sex differentiation begins at the very early crab stage, morphologically represented by the presence of male or female gonopores (although the male gonopores are hardly visible under microscope) and then they develop either male or female pleopods.

On the other hand, G. amboinensis has no such size/sex distribution. Males have not been collected and all the material examined (except megalopae and ovigerous females) bear distinct female gonopores but no typical female pleopods, even in larger specimens up to $C L=5.6 \mathrm{~mm}$. The absence of males in our collection may be the result of sampling bias. No externae or scars of rhizocephalan parasites were 
Table 3. Occurrence of two galatheid symbionts on crinoids found in Okinawa Island. $\mathrm{N}=$ number of crinoids collected; TG = total number of galatheids found; $C=$ number of host crinoids; $\mathrm{G}=$ number of galatheids on a single host; percent occurrence $=$ number of host crinoids/number of crinoids collected.

\begin{tabular}{|c|c|c|c|c|c|}
\hline \multirow[b]{2}{*}{ Crinoids } & \multirow[b]{2}{*}{$\mathrm{N}$} & \multicolumn{2}{|c|}{ G. amboinensis $(\mathrm{n}=32)$} & \multicolumn{2}{|c|}{ G. inflata $(\mathrm{n}=76)$} \\
\hline & & $\mathrm{TG} / \mathrm{C}(\mathrm{G})$ & $\begin{array}{c}\text { Percent } \\
\text { occurrence }\end{array}$ & $\mathrm{TG} / \mathrm{C}(\mathrm{G})$ & $\begin{array}{c}\text { Percent } \\
\text { occurrence }\end{array}$ \\
\hline \multicolumn{6}{|l|}{ Family Comasteridae } \\
\hline Capillaster multiradiatus (Linnaeus) & 46 & $16 / 10(1-3)$ & 21.7 & $11 / 9(1-2)$ & 19.6 \\
\hline Capillaster sentosus (Carpenter) & 2 & & & & \\
\hline Comatella maculata (Carpenter) & 14 & & & $1 / 1(1)$ & 7.1 \\
\hline Comatella nigra (Carpenter) & 8 & $1 / 1(1)$ & 12.5 & $1 / 1(1)$ & 12.5 \\
\hline Comatella stelligera (Carpenter) & 12 & & & $1 / 1(1)$ & 8.3 \\
\hline Comatella sp. & 10 & & & & \\
\hline Comissia magnifica Gislén & 19 & & & & \\
\hline Comanthus alternans (Carpenter) & 34 & & & $1 / 1(1)$ & 2.9 \\
\hline Comanthus gisleni Rowe et al. & 13 & $1 / 1(1)$ & 7.7 & & \\
\hline Comanthus parvicirrus (Müller) & 31 & $2 / 1(2)$ & 3.2 & $32 / 20(1-3)$ & 64.5 \\
\hline Comanthus sp. 1 & 13 & $1 / 1(1)$ & 7.3 & $1 / 1(1)$ & 7.7 \\
\hline Comanthus sp. 2 & 2 & & & & \\
\hline Comanthus sp. 3 & 1 & & & & \\
\hline Comaster schlegelii (Carpenter) & 34 & & & $16 / 12(1-2)$ & 35.3 \\
\hline Comaster nobilis (Carpenter) & 47 & $4 / 4(1)$ & 8.5 & $1 / 1(1)$ & 2.1 \\
\hline Clarkcomanthus littoralis (Carpenter) & 37 & & & $1 / 1(1)$ & 2.7 \\
\hline Oxycomanthus bennetti (Müller) & 32 & & & & \\
\hline Oxycomanthus solaster (A. H. Clark) & 3 & & & & \\
\hline ?Oxycomanthus comanthipinna (Gislén) & 1 & & & & \\
\hline Phanogenia gracilis (Hartlaub) & 44 & $2 / 2(1)$ & 4.5 & $3 / 2(1-2)$ & 4.5 \\
\hline Phanogenia multibrachiata (Carpenter) & 1 & & & & \\
\hline Phanogenia typica Lovén & 1 & & & & \\
\hline \multicolumn{6}{|l|}{ Family Himerometridae } \\
\hline Himerometra bartschi A. H. Clark & 2 & & & & \\
\hline Himerometra robustipinna (Carpenter) & 7 & & & & \\
\hline \multicolumn{6}{|l|}{ Family Mariametridae } \\
\hline Lamprometra palmata (Müller) & 15 & & & & \\
\hline Stephanometra echinus (A. H. Clark) & 3 & & & & \\
\hline Stephanometra indica (Smith) & 16 & & & & \\
\hline Stephanometra spicata (Carpenter) & 31 & $2 / 1(2)$ & 3.2 & $2 / 2(1)$ & 6.5 \\
\hline Stephanometra tenuipinna (Hartlaub) & 2 & & & & \\
\hline Dichrometra spp. & 18 & $2 / 1(2)$ & 5.6 & & \\
\hline \multicolumn{6}{|l|}{ Family Colobometridae } \\
\hline Cenometra bella (Hartlaub) & 2 & & & & \\
\hline Colobometra perspinosa (Carpenter) & 2 & & & & \\
\hline Pontiometra andersoni (Carpenter) & 1 & & & & \\
\hline \multicolumn{6}{|l|}{ Family Tropiometridae } \\
\hline Tropiometra afra macrodiscus (Hara) & 34 & $1 / 1(1)$ & 3.0 & $5 / 5(1)$ & 15.2 \\
\hline \multicolumn{6}{|l|}{ Family Asterometridae } \\
\hline Pterometra venusta A. H. Clark & 3 & & & & \\
\hline \multicolumn{6}{|l|}{ Family Antedonidae } \\
\hline Dorometra nana (Hartlaub) & 2 & & & & \\
\hline
\end{tabular}


observed on the galatheids. It would be of great interest to discover the reason(s) for this sex indeterminate form.

\section{Possible breeding pairs}

Heterosexual pairs of $G$. amboinensis have not been found on the hosts. In $G$. inflata, $26.3 \%$ of galatheid-associated crinoids ( $n=57$ ) held heterosexual pairs, each with a male and an ovigerous female with nearly all (except two) of the ovigerous females larger than their respective partners: in more detail, the pairs were found on $50.0 \%$ of host Comanthus parvicirrus $(\mathrm{n}=20)$, and $33.3 \%$ of host Comaster schlegelii $(\mathrm{n}=12)$ and $50 \%$ of host Phanogenia gracilis $(\mathrm{n}=2)$.

Potts (1915) reported that no pairs were observed. On the other hand, Zmarzly (1984) noted that $50 \%$ of Allogalathea elegans found on Comanthus bennetti (= now Oxycomanthus bennetti) are male-female pairs. Pairs were also recorded for three deepwater species of the galatheidean genera Uroptychus and Munidopsis (see Rice \& Miller, 1992): Uroptychus capillatus Benedict, 1902 on Crinometra brevipinna (Pourtalès), Munidopsis abdominalis (A. Milne Edwards, 1880) on Cidaris blakei (A. Agassiz), $M$. alaminos Pequegnat \& Pequegnat, 1970 on Mesothuria gargantua Diechmann. All the females of the pairs were ovigerous. Permanent or semi-permanent breeding relationships, as suggested by Rice \& Miller (1992), may be presumed for $G$. inflata, but the existence of solitary, non-paired symbionts suggests that new partnerships result from the recruitment individuals of the opposite sex or of sex indeterminate individuals including megalopae.

Galatheid males may have one of two mating strategies: either to guard females until they spawn or to search for other mates. Female attractiveness, to males, could be determined by whether she is about to moult or to release her current brood. Alternatively, females may have one of two spawning patterns: either to delay spawning after mating, thereby increasing the possibility of sperm competition, or to spawn immediately after mating, in which case there is no need for a male to wait around to ensure his paternity. One of the things that would help answer the above arguments would be to have information about the stage of development of each female's brood. If the eggs were in an advanced stage of development we would expect the female to be accompanied by a male, whereas if her eggs had only just been laid then we would expect her to be solitary because a male could spend time more profitably elsewhere. However, examination of the material of G. inflata reveals that the eggs carried by 11 females of 15 heterosexual pairs are light brown in the preservative, suggesting an early stage in development; those of the other four females are eyed. Eggs carried by one of three solitary ovigerous females are eyed, while those of the other two females are not. More data and direct behavioral observations are needed to resolve the mating strategies.

\section{Acknowledgments}

We thank Dr. Ichizo Kogo for his help in identifying the crinoids. Thanks are due to Mr. Tohru Naruse for assistance with fieldwork. Data provided by Dr. Yasushi Fukuda on the development of sex in young Galathea orientalis is greatly acknowledged. The manuscript benefited from reviews by Colin L. McLay of the University of Canterbury and Enrique Macpherson of Centro de Estudios Avanzados de Blanes.

\section{Literature Cited}

Adams, A., \& White, A., 1848. Crustacea. In, Adams, A., The Zoology of the Voyage of H.M.S. Samarang; Under the Command of Captain Sir Edward Belcher, C.B., F.R.A.S., F.G.S., During the Years 18431846. viii +66 pp., 13 pls. Benham and Leeve, London. 
Baba, K., 1979. Expéditions Rumphius II (1975). Crustacés parasites, commensaux, etc. (Th. Monod et R. Serène, éd). VII. Galatheid crustaceans (Decapoda, Anomura). Bulletin du Museum national d'Histoire naturelle, Paris, series 4, section A, 1(3): 643-657.

, 1988. Chirostylid and Galatheid Crustaceans (Decapoda: Anomura) of the "Albatross" Philippine Expedition, 1907-1910. Researches on Crustacea, Special Number 2: $203 \mathrm{pp}$.

Barnard, K. H., 1950. Descriptive catalogue of South African decapod Crustacea. Annals of the South African Museum, 38: 1-837.

Bruce, A. J., 1982. The shrimps associated with Indo-West Pacific echinoderms, with the description of a new species in the genus Periclimenes Costa, 1844 (Crustacea: Pontoniinae). Australian Museum Memoir, 16: 191-216.

Clark, H. L., 1915. The comatulids of Torres St.: with special reference to their habits and reactions. Papers from the Department of Marine Biology of the Carnegie Institution of Washington, 8: 67-125.

Clark, A. H., 1931. A monograph of the existing crinoids. Volume 1 . The comatulids. Part 3. Superfamily Comasterida. Bulletin of the United States National Museum, 82: i-vii, 1-816.

, 1941. A monograph of the existing crinoids. Volume 1. The comatulids. Part 4a. - Superfamily Mariametrida. Bulletin of the United States National Museum, 82: i-vii, 1-603.

- 1947. A monograph of the existing crinoids. Volume 1. The comatulids. Part 4b. - Superfamily Mariametrida (concluded - the family Colobometridae) and Superfamily Tropiometrida (except the families Thalassometridae and Charitometridae). Bulletin of the United States National Museum, 82: i-vii, 1-473.

_, \& Rowe, F. W. E., 1971. Monograph of shallow-water Indo-West Pacific echinoderms. British Museum (Natural History) Publications, 690: 234 pp.

Eeckhaut, I., Deheyn, D., \& Jangoux, M., 1998. Study on the symbiotic fauna of crinoids collected in Hansen Bay (Bismarck Sea, Papua-New Guinea). In: Mooi, R., \& Telford, M. (ed.), Echinoderms: San Francisco. Proceedings of the Ninth International Echinoderm Conference San Francisco/California/USA/5-9 August 1996. p. 151. A. A. Balkema, Rotterdam, Brookfield.
Fabricius, K. E. \& Dale, M. B., 1993. Multispecies associations of symbionts on shallow water crinoids of the central Great Barrier Reef. Coenoses, 8(1): 41-52.

Fishelson, L., 1974. Ecology of the northern Red Sea crinoids and their epi- and endozoic fauna. Marine Biology, 26: 183-192.

Hoggett, A. K. and Rowe, F. W. E., 1986. A reappraisal of the family Comasteridae A. H. Clark, 1908 (Echinodermata: Crinoidea), with the description of a new subfamily and a new genus. Zoological Journal of the Linnean Society, 88: 103-142.

Holthuis, L. B., 1953. Enumeration of the decapod and stomatopod Crustacea from $\mathrm{Pa}$ cific coral islands. Atoll Research Bulletin, 24: 1-66, 2 maps.

Kogo, I., 1998. Crinoids from Japan and its adjacent waters. Special Publications from Osaka Museum of Natural History, 30: 1148.

Lewinsohn, C., 1969. Die Anomuren des Roten Meeres (Crustacea Decapoda: Paguridea, Galatheidea, Hippidea). Zoologische Verhandelingen Uitgegeven door het Rijksmuseum van Natuurlijke Historie te Leiden, 104: 213 pp., 2 pls.

Man, J. D., de, 1888. Bericht ueber die von Herron Dr. J. Brock in Indischen Archipel gesammelten Decapoden und Stomatopoden. Archi für Naturgeschichte, 53(1): 215-600, pls. 7-22a.

Messing, C. G., 1998. Revision of the recent Indo-west pacific comatulid genus Comaster Agassiz. Part 1. The type species of Comaster and Phanogenia Lovén (Echinodermata: Crinoidea: Comasteridae). Invertebrate Taxonomy, 12: 191-209.

Miyake, S., 1938. Galatheids obtained from Osima, Prov. Kii. Annotationes Zoologicae Japonenses, 17:37-42, pl. 2.

Potts, F.A., 1915. The fauna associated with the crinoids of a tropical coral reef with especial reference to its color variations. $\mathrm{Pa}$ pers from the Department of Marine Biology of the Carnegie Institution of Washington, 8: 73-96, pl. 1.

Rice, A. L. \& Miller, J. E., 1991. Chirostylid and galatheid crustacean associates of coelenterates and echinoderms collected from the Johnson-Sea-Link submersible, including a new species of Gastroptychus. Proceedings of the Biological Society of Washington, 104(2): 299-308.

Rowe, F. W. E., Hoggett, A. K., Birtles, R. A., \& Vail, L., 1986. Revision of some comasterid genera from Australia (Echinodermata: Crinoidea), with descriptions of two new 
genera and nine new species. Zoological Journal of Linnean Society, 86: 197-277.

Stimpson, W., 1858. Prodromus descriptionis animalium evertebratorum, quae in Expeditione ad Oceanum Pacificum Septentrionalem a Republica Federata missa, Cadwaladaro Ringgold et Johanne Rodgers Ducibus, observavit et descripsit. Pars VII. Crustacea Anomura. Proceedings of the Academy of Natural Sciences of Philadelphia, 10: 225-252.

Utinomi, H. \& Kogo, I., 1965. On some comatulids from the coastal sea of Kii Peninsula. Publications of the Seto Marine Biological Laboratory, 13(4): 263-286, pl. 12.

, \& 1968. A revised catalogue of crinoids collected from Japanese waters. Proceeding of the Japanese Society of Sys- tematic Zoology, 4: 46-53.

Zmarzly, D. L., 1984. Distribution and ecology of shallow-water crinoids at Enewetak Atoll, Marshall Islands, with an annotated checklist of their symbionts. Pacific Science, 38(2): 105-122.

Addresses: (YF), Department of Chemistry, Biology and Marine Science, University of the Ryukyus, 1 Senbaru, Nishihara-cho, Okinawa 903-0213, Japan; (KB), Faculty of Education, 2-40-1 Kurokami, Kumamoto 8608555, Japan.

E-mails: (YF) k988557@sci.uryukyu.ac.jp; (KB) keiji@gpo.kumamotou.ac.jp 